\title{
Deadly Flood and Landslides amid COVID-19 Crisis: A Public Health Concern for the World's Largest Refugee Camp in Bangladesh
}

\author{
Muhammad Mainuddin Patwary; ${ }^{1,2}$ ( Alfonso J. Rodriguez-Morales ${ }^{3,4,5}$
}

1. Environment and Sustainability Research Initiative, Khulna, Bangladesh

2. Environmental Science Discipline, Life Science School, Khulna University, Khulna, Bangladesh

3. Grupo de Investigación Biomedicina, Faculty of Medicine, Fundacion Universitaria Autónoma de las Américas, Pereira, Colombia

4. Master of Clinical Epidemiology and Biostatistics, Universidad Cientifica del Sur, Lima, Peru

5. School of Medicine, Universidad Privada Franz Tamayo, Cochabamba, Bolivia

\section{Correspondence:}

Muhammad Mainuddin Patwary

Environment and Sustainability Research Initiative

Khulna 9208 Bangladesh

E-mail: raju.es111012@gmail.com

Conflicts of interest/funding: This research did not receive any financial support. The authors declare no conflict of interest.

Keywords: Bangladesh; climate change; COVID-19; natural disaster; Rohingya refugee

\section{Abbreviations:}

COVID-19: coronavirus disease 2019

SARS-CoV-2: severe acute respiratory syndrome coronavirus 2

Received: November 5, 2021

Accepted: November 12, 2021

\section{doi:10.1017/S1049023X22000164}

(C) The Author(s), 2022. Published by

Cambridge University Press on behalf of the

World Association for Disaster and Emergency Medicine.
Patwary MM, Rodriguez-Morales AJ. Deadly flood and landslides amid COVID-19 crisis: a public health concern for the world's largest refugee camp in Bangladesh. Prehosp Disaster Med. 2022;37(2):292-293.

Since the beginning of 2020, the severe acute respiratory syndrome coronavirus 2 (SARS$\mathrm{CoV}-2$ )/coronavirus disease 2019 (COVID-19) pandemic brought unprecedented risk to global public health and devastated the global health system. In the absence of an available vaccine, the world has already witnessed multiple waves of this pandemic. Since the first case was recorded there on March 8, 2020, Bangladesh has endured eighteen months of infection without reaching zero cases. ${ }^{1}$ The country is now experiencing a devastating second wave of the pandemic, caused mainly by the coronavirus Delta strain, which was first identified in Bangladesh on May 8, 2021 through a traveler from India. ${ }^{2}$ Additionally, Hasan reported that the Delta variant caused $98 \%$ of detected COVID-19 cases during the second wave of the pandemic in Bangladesh. ${ }^{3}$

The current pandemic is particularly vulnerable to marginalized people (refugees) in lowmiddle income countries because of their poor living conditions and limited access to washing, sanitation, and hygiene facilities. The country has hosted one million Rohingya refugees residing in 34 camps in Cox's Bazar district who fled away from Myanmar on August 25, 2017. The highly transmissible Delta strain is causing an infection epidemic throughout Bangladesh. Approximately 20,000 infections and 250 deaths were recorded in Cox's Bazar district, which borders Myanmar and is home to 34 refugee camps. As of September 19, 2021, 3,026 cases were reported, along with 32 reported deaths, representing a crude fatality rate of $1.1 \%$ in the Rohingya refugee camp. ${ }^{5}$ Numerous factors, including prior sexual assault, inactivity, and substandard living circumstances, with ten or more people per space, make the Rohingya population vulnerable to non-communicable illnesses. In addition, thousands of older people and children live in the area. Many of them suffer from non-communicable and chronic illnesses that render them vulnerable to viruses. Thus, implementing preventive measures (eg, social distance) is difficult for Rohingya refugees living in congested camps (40,000 people per square kilometer). ${ }^{6,7}$

While the country struggles to cope with the increasing COVID-19 pandemic, the Rohingya camp in Cox's Bazar is further exacerbated by the negative impact of monsoon weather. The camp experienced over 300mm rainfall from July 27 - August 1, 2021, the highest ever in the last 20 years. ${ }^{8}$ Additionally, the Cox's Bazar has experienced over $1300 \mathrm{~mm}$ of rainfall from July 27 - September 1, 2021; over 200mm just from August 27-28. These days of heavy monsoon rains and strong winds pelted massive refugee sites, causing deadly flash floods and life-threatening landslides. The flood and landslide caused heavy damage to shelters, injured refugees, and impeded aid response by blocking them from accessing camp. As a result, 87,617 refugees were affected, 25,469 displaced, and ten refugees have died in the floods and landslides. In addition, 3,564 refugee shelters were partially damaged, 3,065 severely damaged, 2,489 fully damaged, and 15 were destroyed. ${ }^{9}$ The situation has further worsened by the COVID-19 crisis since the country has undergone a nation-wide lockdown to halt the transmission of virus infection.

Severe health consequences could occur through direct contact with floodwater. It has been estimated that floods, landslides, and cyclonic hazards caused the direct death of approximately 658,770 people from 1970 through 2017 in Bangladesh. ${ }^{10}$ However, the indirect effect of a flood is mainly unknown. Due to disruption in health services and lack of social support system, there is an increasing risk of further exacerbating people's illness with cardiovascular diseases, cancers, chronic respiratory diseases, and diabetes after flooding. ${ }^{11}$ Importantly, it is expected that communicable and emerging diseases, including 
leptospirosis, malaria, cholera, gastrointestinal diseases, dermatological disease, and a substantial increase in psychological disorder could appear after flooding, in particular, the area with the poor hygienic condition and population displacement. In addition, direct contact with contaminated floodwater could cause eye and skin infection. Flood events are also responsible for triggering vector-borne diseases in endemic areas. For example, severe flooding in Peru in 2017 was linked with a significant dengue and chikungunya epidemic, with $>19,000$ dengue cases. ${ }^{3}$ Since the severe floods devastated the refugee camp, the unsanitary conditions and risks of water-borne disease transmission, considering hundreds of acute watery diarrhea cases already reported in the camp, could be disastrous for Rohingya refugees. ${ }^{4}$ Further, the Rohingya themselves have resisted moving to shelters that exacerbated the crisis due to their concerns about privacy, space, and loss of shelter plots, as well as disease transmission (COVID-19 and diarrhea in particular). However, approximately 5,000 refugee families were temporarily relocated to other community shelters. Thus, evacuating the people in safe places where a lack of maintaining proper social distancing could be another hub of source of SARS-COV-2 infection. Concurrent occurrence of natural disaster and COVID-19 crisis has placed an additional strain on the already fragile health care system in
Bangladesh. In this uncertain period, the increasing health demand on an already vulnerable health care system, COVID-19, emerging infections, emerging diseases, and two natural disasters transform the situations into a humanitarian crisis.

Considering the situation, the Government of Bangladesh and the international humanitarian organizations should respond quickly, assertively, and provide life-saving assistance and support to Rohingya refugees. Notably, the floods and the likely COVID19 comeback should remind us not to give up, strengthen biosecurity measures, and offer social support to the refugee. Although Bangladesh and the international humanitarian community take some initiatives to reduce the immediate impact, this is insufficient. ${ }^{9}$ Therefore, the Bangladesh Government, nongovernmental organizations, and the International Humanitarian Community should scale up their efforts to avoid further catastrophe. Under the nation-wide vaccination program, a total of 37,000 Rohingya refugees have been vaccinated util now, which could be a hope for reducing infection. However, natural disasters, lack of space, and permanent shelters remain an issue for refugees. Thus, an integrated action plan is needed and must be implemented immediately to reduce the risk of increased infection and reduce the impact of natural disasters.

\section{References}

1. Patwary MM, Hossain MR, Shuvo FK, Ashraf S, Sultana R, Alam MA. Protecting sanitation workers in low-middle income countries amid COVID-19. Ann Work Expo Health. 2021;65(4):492-493.

2. Moona AA, Daria S, Asaduzzaman M, Islam MR. Bangladesh reported delta variant of coronavirus among its citizen: actionable items to tackle the potential massive third wave. Infect Prev Pract. 2021;3(3):100159.

3. Hasan M. BSMMU: $98 \%$ of Bangladesh's Covid patients infected with Delta variant. Dhaka Tribune. 2021. https://www.dhakatribune.com/bangladesh/2021/08/05/bsmmu98-of-bangladesh-s-covid-patients-are-infected-with-delta-variant. Accessed November 4, 2021.

4. The Lancet. The Rohingya people: past, present, and future. Lancet. 2019; 394(10216):2202.

5. World Health Organization. Coronavirus Disease 2019 (COVID-19). (13-19 September 2021) UPDATE in Cox's Bazar. 2021. https://www.who.int/. Accessed November 4, 2021.

6. Banik R, Rahman M, Hossain MM, Sikder MT, Gozal D. COVID-19 pandemic and Rohingya refugees in Bangladesh: what are the major concerns? Glob Public Health. 2020;15(10):1578-1581.
7. Jubayer F, Kayshar S, Limon TI. First COVID-19 case in the Rohingya camp in Bangladesh: needs proper attention. Public Health. 2021;191:20.

8. Al Jazeera. Through refugee eyes: Deadly floods in Bangladesh Rohingya camps. Gallery News, Al Jazeera. 2021. https:/www.aljazeera.com/gallery/2021/8/3/throughrefugee-eyes-fatal-floods-in-bangladeshs-rohingya-camps\#: :text=It $\% 20 \mathrm{has} \% 20$ not $\%$ 20 stopped $\% 20$ raining,the $\% 20$ world's $\% 20$ largest $\% 20$ refugee $\% 20$ camp.\&text $=$ The $\%$ 20floods $\% 20$ damaged $\% 20$ temporary $\% 20$ shelters, refugees $\% 2 \mathrm{C} \% 20$ three $\% 20$ of $\% 20$ them \%20children. Accessed November 4, 2021.

9. Inter-Sector Coordination. ISCG Monsoon Response Flash Update \#6, 1 September 2021. Humanitarian Response. https:/www.humanitarianresponse.info/en/operations/ bangladesh/document/iscg-monsoon-response-flash-update-6-1-september-2021. Accessed November 4, 2021.

10. Matsuyama A, Khan FA, Khalequzzaman M. Bangladesh Public Health Issues and Implications to Flood Risk Reduction. In: Public Health and Disasters. Springer Singapore; 2020.

11. Ryan B, Franklin RC, Burkle FM, et al. Identifying and describing the impact of cyclone, storm and flood related disasters on treatment management, care and exacerbations of non-communicable diseases and the implications for public health. PLoS Curr. 2015;7:ecurrents.dis. 